\title{
On the Space-depiction Techniques of Chinese Traditional Architectural Drawings
}

\author{
Peng Peng \\ College of Architecture and Urban-Rural Planning \\ Sichuan Agricultural University \\ Chengdu, China
}

\begin{abstract}
Ruled-line painting is the best foundation of research on ancient Chinese buildings. From existing ruled-line painting works, it can be figured out that ruled-line paintings of the Northern Song Dynasty have had a great breakthrough based on predecessors in the respect of manifestation with regard to sense of space. A group of experienced painters of ruled-line painting at that time such as Zhang Zeduan, Guo Zhongshu, Yan Wengui and the like know a lot about "subtle principles" of "Same Proportion as Material Objects" and "Hundreds of Diagonals Gathered to One Point", whose works' sense of volume, sense of space and sense of stability are universally superior to those of predecessors. Therefore, a form or framework of ancient architectural drawings basically took shape at that time. In this paper, analyses on spacedepiction techniques of Chinese traditional architectural drawings before the introduction of Western perspective theory are done through studying ruled-line paintings of Song Dynasty.
\end{abstract}

Keywords-architectural drawing; ruled-line painting; Hundreds of Diagonals Gathered to One Point; Viewing the Small from the Whole Perspective

\section{INTRODUCTION}

Architectural drawing, as its name implies, belongs to the painting category with buildings as drawing objects. During the Wei and Jin Dynasties, painting with buildings as main subjects was called painting of terraced building, just as what $\mathrm{Gu}$ Kaizhi of the Eastern Jin Dynasty said in Introduction of Famous Paintings of Wei and Jin Dynasties: "Though buildings like terraced buildings and so on are complex, it is easy to copy their shapes with wonderful effects, which can be achieved without "Transformation in Imagination Brings Unexpected Acquisition." It is also known as painting of "wooden house", painting of "balcony", painting of "palace" and so forth in the painting history and painting theory of Tang and Song Dynasties. As what "Concerning figures, dress, carriages and horses and balconies, it owns essence of painting techniques both in the southern part and northern part." and "Among painting branches, painting of figures is the most difficult, painting of animals takes the second place, painting of landscapes the third and painting of buildings the last." mentions, architectural drawing became an independent category in the Song Dynasty. In the period of Emperor Huizong in Song Dynasty, painting philosophy was established with six branches, namely Buddhism, figures, landscapes, animals, plants and wooden houses. Since the
Yuan Dynasty, the word "ruled-line painting" has been widely used and accepted as well as become the painting branch which takes buildings as subjects. In Notes of Nan Village of Tao Zongyi, painting is divided into 13 categories, among which "ruled-line painting of buildings" ranks the eighth as the drawing majoring in building subjects such as palaces, towers, houses and the like. Meanwhile, in Appreciation and Evaluation of Paintings, Tang Hou said: "When it comes to paintings, we will say that paintings have 12 categories with landscape painting taking the lead and ruled-line painting at the bottom." Moreover, ruled-line painting is particular about accuracy of buildings' shape and structure and lay emphasis on shaping sense of space. "Drawing of Immortals' Buildings by Guo Zhongshu" recorded in Painting and Calligraphy Album of Peiwenzhai gives us a feeling that we can walk into the painting and open and close its handrails, doors as well as windows, which reflects that architectural drawing at that time has achieved a great progress in the aspects regarding stereoscopic impression and sense of space. As for how to create buildings' 3D sense of space on a 2D plane to achieve effect of "Feeling like Being Personally on the Scene", just as what Guo Ruoxu of the Song Dynasty said in part one "Talk on Model Production" of Overview of Painting: "Drawing wooden houses should be done according to the same proportion as material objects with strong and fluent strokes and by gathering hundreds of diagonals to one point (parallel perspective) to create sense of space. Taking famous painters like Guo Zhongshu and Wang Shiyuan from the time before the Sui, Tang and Five Dynasties to the beginning of the founding concerning our country as examples, buildings in their paintings, whose four cornices and dougongs (a system of brackets in Chinese building; wooden square blocks inserted between the top of a column and a crossbeam.) can be seen clearly, are drawn with clear and well-balanced strokes in accordance with precise proportions, which quite conforms to rules.", it is necessary to obey "Same Proportion as Material Objects", "Hundreds diagonals gathered to one point (parallel perspective)" and "One Diagonal Followed by Hundreds of Diagonals". However, here come the questions: What kind of perspective techniques are "Same Proportion as Material Objects", "Hundreds diagonals gathered to one point (angular perspective)" and "One Diagonal Followed by Hundreds of Diagonals" specifically representing? What "rules" do they 
conform to? There are no more written records in ancient books.

\section{MUlti-VIEWPOINT OBSERVATION AND DEPICTION TECHNIQUES}

As we all know, principles of perspective are established on the premise of building points of sight. According to painting traditions, "Focus Perspective" is usually used in Western paintings, that is, an observer fixes a viewpoint and accurately records things within his field of vision. Though this kind of perspective rule has been referred to in theory of ancient Chinese painting for many times, there is basically no spatial processing techniques concerning focus perspective appearing in Chinese traditional drawings including the ruled-line paintings which can show spatial relationship in the best way. The classic work of ruled-line painting in the Song Dynasty, Along the River During the Qingming Festival, is drawn by adopting the perspective angle of looking down from high place and moving point of sight to break the restrictions of visual range. It makes everyone feel like being guided to walk into the wharf of Bian River and street along the river from the suburbs of Bianjing (Municipality of Kaifeng today), then we reach the Rainbow Bridge of the Bian River and continue walking into city along the main road at this end of the bridge, and finally we stop in the downtown street, which is just the feeling that we are personally in this place for living and travelling. Meanwhile, this kind of observation method is mentioned by Guoxi of the Song Dynasty in The Lofty Message of Forest and Streams: "Mountains' shapes in my eyes vary with changes of the distance between me and the mountains, that is, mountains' shapes vary with steps. Moreover, mountains' shapes vary with changes of the side we chose to face, that is, mountains' shapes vary with sides. Since a mountain has hundreds of shapes, it is hard to know clearly what on earth the mountain is like." To distinguish Chinese painting from Western painting in perspective technique, this kind of observation methods, namely "Mountains' Shapes Vary with Steps" and "Mountains' Shapes Vary with Sides", is called scattered-point perspective. This book also mentions: "Eyes' ability to appreciate sceneries is limited, so it is impossible to see all the sights ( for example, it is impossible for us to see ideal places like "Fantasyland", "Mount Fangzhang" and so forth with eyes. ) To make up defects of our eyes, landscape existing in imagination is copied with writing brushes. With part of the imagined landscape is drawn on papers, imagined landscape becomes something that eyes can see." Due to the limited vision of human beings, there is no way to observe scenery from a fixed point of sight in an overall and complete way. Therefore, painters' viewpoint of traditional Chinese painting does not have to be fixed in one place and restricted by fixed range of vision. Instead, viewpoint can be moved as required. During the painting, painters can freely put things observed from different viewpoints together in line with their own needs of creation to obtain various atmospheres they want to create. There is no doubt that it is extraordinarily difficult to create such a magnificent, busy and lively scene just relying on painters' actual observation. However, special observation methods in Chinese paintings, namely "moving-point perspective" and "movement of steps with shift of scenes", have almost perfectly solved this problem. Based on perspective rules, figures overlooked from high visual angel transform quite a lot, so multiviewpoints are often used in ancient rule-line paintings to put figures of eye level into the background which is slightly overlooked. For instance, in Along the River During the Qingming Festival, all figures appear as the images obtained from eye-level angle and with similar sizes as well as proportions, no matter they are sitting or standing and they are far or near. This problem is also talked about in Pan Tianshou's Theory of Painting: “ By using bird's-eye perspective with an angle similar to $45^{\circ}$, long, straight and narrow garden are drawn and arranged, which makes sceneries layered and have depths. However, this kind of perspective will cut the length of figures, which makes figures different from those from eye-level perspective and makes viewers uncomfortable. Therefore, painters of our country also put eye-level figures to the layout done through perspective of bird's-eye view, which not only enables landscape to be layered but keeps figures in similar shape to daily images." Since figures observed from high place are quite different from those of daily life, eye-level angle is always used to process images such as figures and carriages and horses, which, despite figures' position in the picture, enables figures to mostly appear in the eye-level state without changes caused by looking up or looking down. If careful observations are conducted, it is also easy to discover that steps and stairs of buildings in ruled-line paintings of the Song Dynasty are always processed as wide at the top and narrow at the bottom as if we are looking down from the steps in the drawing. Meanwhile, in works of ruled-line painting, nearly all tables and chairs, wooden benches, beds and furnishings are processed in the light of this way and take on the inverse perspective effect of "the farther, the longer". The reason, for my part, is related to painting habits mentioned before. For there is no fixed viewpoint, images obtained from various viewpoints are gathered together. We can make a hypothesis that the painter enters the drawing to finish it as if he is looking down by standing on the steps instead of looking at the drawing from the viewpoint of a viewer as what focus perspective says as shown in "Fig. 1".
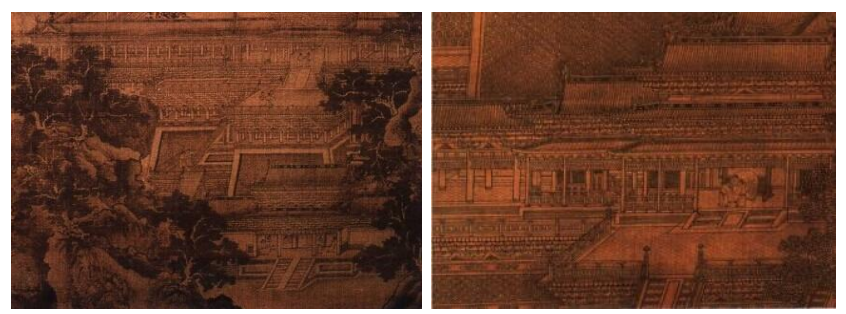

Fig. 1. Song Dynasty, Along the River During the Qingming Festival.

At this moment, "the farther, the longer" appearing in the drawing is just "the farther, the shorter" which is felt by the painter by staying in the drawing as shown in "Fig. 2". 

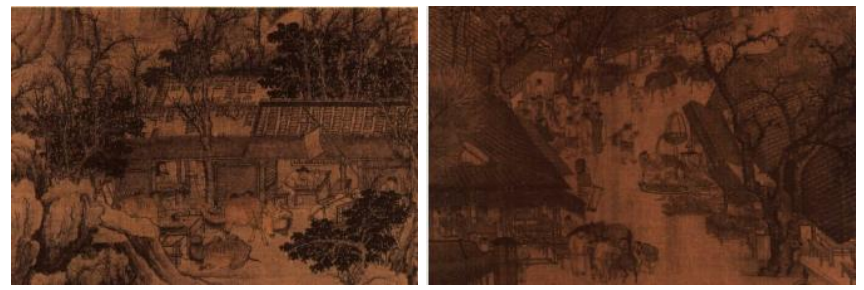

Fig. 2. Song Dynasty, Along the River During the Qingming Festival.

There are not many reasons for this behavior, because it is just a simple artistic processing conducted by painters of ruled-line drawing according to the most direct visual perception. Besides, in the works of ruled painting, when there is a certain angle between buildings and observers, roofs will take on the phenomena of "the farther, the wider" and " the farther, the longer" which gives us the feeling that roofs are lifted up with an angle and bird's-eye effect is strengthened as shown in "Fig. 3".

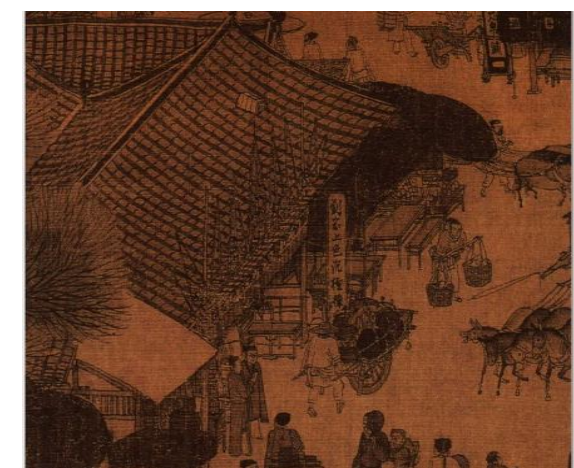

Fig. 3. Song Dynasty, Along the River During the Qingming Festival.

\section{PARALLEL-PERSPECTIVE PAINTING TECHNIQUE OF "HundREDS OF DiAgONALS GATHERED TO ONE POINT"}

Once range of vision is determined, we should consider the extension direction of the scenery we see, namely the matter of spatial depth. In theory of Western focus perspective, the extension direction of spatial lines is determined through vanishing points. According to the principle of the "the farther, the smaller", the lines in " $3 \mathrm{D}$ " space which are originally parallel to the picture are still parallel in the picture while the lines which are not parallel with the picture are gathered to one point, namely the vanishing point. Based on the number of vanishing points, Western perspective techniques are divided into three categories: parallel perspective (one vanishing point), angular perspective (two vanishing points) and oblique perspective (three vanishing points). Different from focus perspective, the pursuit of Chinese people towards spatial depth is based on parallel method. Traditional architectural drawing is done by using a kind of quite casual graphing method concerning parallel lines without vanishing points, whose main characteristic is that all perspective lines in the painting are parallel instead of being gathered to one vanishing point. Guo Zhongshu's Painting on the Summer Palace of Emperor Xuanzong of the Tang dynasty is an outstanding representative work of architectural drawing regarding palace, which is shown in "Fig. 4".

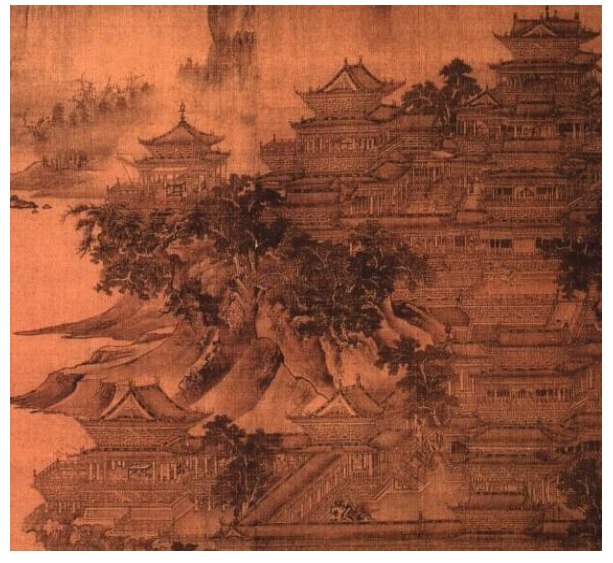

Fig. 4. Guo Zhongshu, local "Ming Huang summer palace map"

The Summer Palace faces river with mountains on the back. Mountain ridges take up one third of the drawing while foreground building groups takes up two thirds. When it comes to perspective, almost all buildings are depicted from the angle of overlooking and slightly inclined left or right of the front side. All the lines vertical to the drawing are in parallel instead of gathering inward. Moreover, eaves of buildings' side face, top and bottom lines of doors and windows, foundation lines and the like all extend as parallel lines with the same slope as well as have no vanishing point. For the harmony and unity of the painting, the lines of the same group of buildings which are vertical to the picture own almost same inclined angles, unnatural transition parts between nearby and far-away buildings are covered and linked with mist, trees and stones of unobvious perspective, and storey heights of all the buildings in building groups are basically identical rather than changing with positions of buildings. Accordingly, "Hundreds of Diagonals Gathered to One Point" and "One Diagonal Followed by Hundreds of Diagonals" said in the ancient painting theory, to my mind, just refer to the above-mentioned method in which the lines of showing depth are processed into oblique parallel lines, based on which "Same Proportion as Material Objects" and "Paint buildings according to same proportion and specific standards with little deviations" in ruled paintings of the Song Dynasty are developed. Unlike techniques such as focus perspective and so on which enables angel of inclination and sizes to be determined on the basis of geometric graphing method, this kind of perspective technique does not have quantifiable standards for length and slope of the third dimension's lines, which, instead, are determined in the light of painters' personal experience. Generally speaking, the oblique angle mostly ranges from $30^{\circ}$ to $60^{\circ}$, among which $45^{\circ}$ is the most common. As for proportion, the length of the front side is scaled down in terms of the original buildings' proportion while the length in the direction of depth is roughly scaled down in accordance with half of the proportion regarding the original buildings. In Art and Visual Perception of Arnheim, this kind of technique is defined as "isometric perspective", which can be divided into "isometric perspective of the front" and 
"isometric perspective of oblique plane", as shown in "Fig. 5 ".

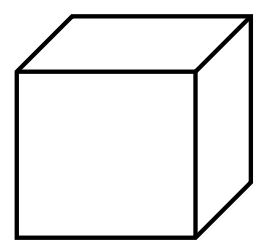

Fig. 5. isometric perspective of the front.

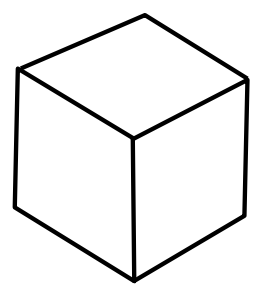

Fig. 6. isometric perspective of oblique plane.

When in perspective of the front, one plane of a 3D object is a vertical front in the picture and all the lines of the third dimension which are vertical to the front intersect the plane with oblique angles, which are all the same to make all the lines of the third dimension in parallel. Then this perspective is called "isometric perspective of the front".Distinct from "isometric perspective of the front", "isometric perspective of oblique plane" have some simple changes towards the first one's frame structure. In this perspective, either of two axes' directions of the objects has certain angles with the plane of the drawing in front of viewers, every plane surface has transformation and shortening, and its space owns certain slope, which makes the painting quite dynamic. When talking about these two forms of isometric perspectives, Arnheim mentioned that "isometric perspective of the front" possesses great superiority: this kind of perspective is a way to reproduce the depth of space by combining typical "front side" with "side plane" which is tilted and shortened. It displays the 3D effect with the minimum transformation as well as keeps many objective characteristics of original buildings. No matter "the front" represents the front elevation or side elevation, this kind of perspective maintains original shape and proportion. What's more, those lines which gradually extend to the depth of space also keep their objective proportion. Frame structure of the space drawn in this way is same as that of the space where viewers stay, which is relatively stable and motionless in the work. Besides, this kind of perspective also reduces the uncertainty of the object caused by perspective transformation as well as maintains the realness of the "known" object. He said: "this form is not used for the replication of physical cubes but to reproduce objects naturally through media of presentation. Since the easiest perceptual structure and orientation are applied to it, paintings of this kind of perspective are not only easy to draw, but also easy to understand." The "isometric perspective of the front" enjoys great popularity among painters of ruled-line painting for it not merely meets visual requirements but also precision requirements of "Same Proportion as Material Objects" with its quantifiable characteristic. Therefore, this kind of perspective is widely used in ruled-line painting works of the Northern Song Dynasty as well as becomes the mainstream depiction technique in the reproduction of ancient Chinese buildings. On the contrary, all the planes in paintings of "isometric perspective of oblique plane" are tilted compared with the picture. It seems that painters draw paintings by observing the inside from a relatively peripheral space far away from the ground, which brings feelings of dynamic and uncertainty. Usually, this form is suitable for dramatic scenes and only accounts for a little percentage in ruled-line painting works. It is restricted to some special occasions or used as means to change and adjust composition of the painting.

\section{PICTURE-COMPOSING \& VIEW-SEEKING MODE OF}

\section{"VIEWING THE SMALl FROM THE WHOLE PERSPECTIVE"}

In the Dream Pool Essays, Shen Kuo of Song Dynasty expressed his opinions on the way to compose paintings and seeking views concerning Chinese landscape paintings: "Paintings of Li Cheng with regard to pavilions, towers and so on are all drawn by looking up, namely "Cornices Drawn by Looking Up", which means that a person can see a building' rafter by looking up if he is in a position as high as the bottom of a building. Actually, it is wrong. Observation method towards landscape is often that viewing the small from the whole perspective, which is just like a person looking at artificial hills. If we only want to depict the real visual sense, "seeing", of a person in the real landscape and draw paintings by looking up from a low position, you can only see cornices when you put your eyes on buildings and you can only see one mountain when you look at mountains, which absolutely hinders viewers to appreciate rolling mountains as well as vertically and horizontally distributed ravines, let alone everything in the courtyards and back alleys. On all accounts, it is impossible to draw these things mentioned above according to Li Cheng's method." In this paragraph, Shen Kuo clearly shows his negative attitude towards the phenomenon of "Cornices Drawn by Looking Up" appearing in landscape painting caused by valuing people's real visual sense in real landscape. Just like what Shen Kuo said, if we stubbornly stick to the real visual sense in real landscape and confine ourselves within fixed range of vision, there will never exist "rolling mountains", "vertically and horizontally distributed ravines", "everything in courtyards and back alleys" and the like in landscape paintings, which means the loss of richness concerning painting and feeling of being personally on the scene of the landscape painting. "Viewing the Small from the Whole Perspective" is just the summary of the unique way of thinking towards Chinese landscape painting, that is, treating real landscapes as artificial hills and bonsais and observing as well as depicting them in an all-around and multiperspective way. The form of art presentation, using "what we know" to portray "what we have seen", is always adopted in ancient Chinese painting. In other words, Chinese painters of landscape painting seldom paint from nature like drawing Western landscape paintings. Instead, they finish painting on the basis of daily travels and experiences. In most cases, the painting objects are not confined within one fixed viewpoint 
and specific range of vision and are drawn through moving viewpoint. After overall observation, the painting of natural scenery is created via subject thinking and feeling. In such a circumstance, artists acquire sufficient freedom of creation to create objects in their mind by using imagination without considering the location for "copying", which totally differs from the copying passion of Western People towards "what they have seen". At first, ruled-line painting emerges as the background of figure painting or landscape painting. Therefore it naturally inherits landscape painting's way of thinking concerning the choice of visual angle. The so-called "the farther, the longer" is just the space-processing method of pushing away and pulling closer objects in a relatively casual way, which is not restricted by fixed viewpoints and enables painters to master objects in a dynamic and overall way. Just as what Guo Xi said, "Referring to landscape, only if a person appreciate it in a distance can he have a panoramic view of the landscape including its various conditions", "Viewing the Small from the Whole Perspective", namely the perspective requirements of long sight distance, is an significant macroscopic observation method adopted by Chinese painting. For instance, for the long scroll like Along the River During the Qingming Festival whose scenes are usually quite big, it is hard to find a unified point of sight. But under the impacts of movingpoint perspective concerning "movement of steps with shift of scenes", every part of the painting has its own visual focus and there are natural transitions among various parts: they are linked with long bridges, rivers, or trees; With the constant changing of viewers' lines of sight, continuous landscapes appear and natural transformation of space takes place; Painters can continue drawing this long scroll all the time without considering the matter of harmony. Furthermore, viewing and appreciating habit regarding traditional Chinese paintings is appreciating a painting part by part and moving point of sight gradually. So landscapes of different parts in the painting will not emerge in viewers' eyes at the same time. This is the processing of spatial relationship in the transverse direction while in the longitudinal direction, positions of drawing objects in the painting indicate actual location of objects in real space. Overlaps among buildings in the front and back should be avoided to the greatest extent, that is, the farther buildings are, the higher should buildings be paint in the painting. Through drawing the picture layer by layer, all kinds of varied layouts, complexly distributed courtyard groups and all the single buildings are unfolded before viewers' eyes. Apart from this kind of observation method of long sight distance which is similar to bird's-eye view, the distant views is often painted as the close views in the ruled-line painting. For example, in Along the River During the Qingming Festival, the indoor scenes of buildings and all kinds of words on horizontal tablets and couplets upon doorposts which are supposed to be blurred are extremely distinct; in the part of Rainbow Bridge, structure and shape of the "Rainbow Bridge" are accurately and subtly depicted while figures on the bridge and boats are vividly portrayed with clear beards and eyebrows as well as expressions.

\section{CONCLUSION}

To sum up, perspective techniques of Chinese traditional architectural paintings represented by ruled-line paintings totally differ from those of Western Paintings which reflects two kinds of completely different aesthetic tastes. In the processing of spatial relationship, ruled-line paintings of the Northern Song Dynasty have formed a mode: Under the perspective requirements of "Hundreds of Diagonals Gathered to One Point" and "Same Proportion as Material Objects", space should be depicted with parallel lines as many as possible and beauty of buildings should be presented through a bird's-eye view in a distance. Meanwhile, with the help of ruled-painting of the Northern Song Dynasty, a whole set of perspective rules and depiction techniques conforming to our national appreciating habits have been figured out, especially the nearly stylized depiction technique for buildings' space depiction. Though accuracy of the "proportion" used by ancient people in the ruled-painting needs further study, this kind of depiction technique of space owns incomparable advantage over the focus perspective in many ways. Picture-composing and view-seeking way of "Viewing the Small from the Whole Perspective" and "the Farther, the Higher" breaks resrtictions of time and space as well as makes the space of Chinese traditional architectural painting pretty dynamic. Moreover, painters also break the limitation of viewpoint and integrate visual impressions of multiple space together through "Looking Up and Bird's-eye View" to depict everything in the painting, which gives viewers a sense of being personally on the scene.

\section{REFERENCES}

[1] Lu Fusheng. History of Chinese painting: ruled-line paintings of buildings [M]. Shanghai: Shanghai Fine Arts Publisher, 2006.

[2] Li Lina. The Beauty of palaces and pavilions: exhibition of ruled line painting [M]. Taipei: National Palace Museum, the Republic of China 89.01.

[3] (the Five Dynasties) Guo Zhongshu. Guo Zhongshu's painting of summer palace [M]. Shanghai: Shanghai Ancient Book Publishing House, 2003

[4] Zhou Jiyin. Summary of Chinese painting theory [M]. Nanjing, Phonix Fine Arts Publishing. LTD, 2005.

[5] (US) Rudolf A; Art and Visual Perception [M], translated by Teng Shouyao and Zhu Jiangyuan. Chengdu: Sichuan People's Publishing House, 1998

[6] Tang Qi. Chinese painting theory of past dynasties [M]. Wuhan: Hubei Fine Arts Publishing House, 2009.12.

[7] Yu Anlan. Painting Series [M].Shanghai: Shanghai Fine Arts Pulishing House, 1982.

[8] Pan Tianshou. Notes on painting of Cloud Pavilion[M]. Shanghai: Shanghai People's Publishing House, 1980.

[9] Liu Dunzhen. History of ancient Chinese architecture [M]. China Architecture \& Building Press, 1984.

[10] Fu Dongguang. Buildings in the painting -- ancient Chinese architectural painting collected by National Palace Museum [J]. Palace Museum Journal, 1999.

[11] Fu Xinian . Ancient Chinese architectural drawing [J]. Cultural relic, 1998.

[12] Wang Xuedan. Research on ruled-line painting of the Song Dynasty [D]. Master's thesis, Southwest University, 2002. 